\title{
Consensus analysis of Multi-agent Systems under switching topologies by a topology-dependent average dwell time approach
}

\author{
Dianhao Zheng, Hongbin Zhang *, Qunxian Zheng
}

School of Electronic Engineering, University of Electronic Science and Technology of China, Chengdu 611731, People's Republic of China.

*zhanghb@uestc.edu.cn

\begin{abstract}
This paper addresses the consensus problem for a class of any order multi-agent systems under switching topologies which could include kinds of unconsensusable topologies. The consensus problem, depending on structure properties and the corresponding topology, is researched with fixed structure properties under directed switching topologies. By properties of Laplacian matrix, the consensus problem for multi-agent systems is converted into the stability problem of the corresponding switched systems with a Laplacian-like matrix. Some sufficient conditions for consensus are presented by using the dwell time approach. Finally, numerical examples and the results of computer simulation are given to verify the theoretical analysis.
\end{abstract}

\section{Introduction}

Distributed cooperative control of multi-agent systems has received considerable attention over the past few years due to the rapid developments of computer science and its wide application in many areas, such as industrial production [1], sensor networks [2], vehicle systems [3], and spacecraft flying [4]. The fundamental tasks of cooperative control have been widely investigated, e.g. consensus [5-8], tracking control [9], containment control [10] [11], flocking [12], rendezvous [13]), and formation control [14]. In order to study and analyze these fundamental tasks, matrix theories, graph theories, control theories and some other research approaches and theories have 
been employed [15] [16].

Consensus, as a kind of cooperative behavior of multi-agent systems, has been researched a lot in recent years $[5-8,17,18]$. To make all agents converge to a common state was the major objective of consensus for multi-agent systems [8]. The common state was called the group decision value in [5]. The group decision value was not decided by centralized systems, but by each agent using the local information of its own and neighbors.

Some preliminary results about the first-order consensus under fixed and switching topologies have been reported in $[5,6,15,33,35-37]$. In [6], it was shown that the first-order consensus can be achieved asymptotically if the union of the directed interaction graphs contained a spanning tree frequently enough. The first-order asynchronous consensus problem under directed switching topologies was shown to be solvable if the union of the topologies contained a spanning tree in [35]. First-order leader-following consensus under undirected switching topologies was researched in [36] [37], and consensus tracking under directed switching topologies in [43], showing that the joint connectivity was a key condition to ensure the leader-following consensus.

Some conclusions about the second-order consensus have been reported in [19-23, 34, 38]. In [19], some preliminary schemes for the second-order consensus have been studied, and it has been reported that the second-order consensus may fail to be reached even if the network topology had a directed spanning tree, i.e., consensus required other conditions besides having a spanning tree. The second-order consensus was affected by more factors than the first-order consensus. For the same agents and initial conditions, even a consensus could be reached under some fixed topologies (called consensusable topologies), the consensus could not be achieved under other topologies (called unconsensusable topologies). The unconsensusable topologies resulted from many factors, such as improper gains (coupling strengths) or no-spanning tree. In order to further study these consensus conditions, the problem about second-order consensus in multi-agent dynamical systems under fixed topologies was discussed, and some conditions were given in [20-22]. In addition, second-order consensus under switching topologies with balanced networks was discussed in [23]. The second-order leader-following consensus was studied in [38], under the assumption that the leader was fixed for switching topologies. 
Some early results for the consensus of the three-order and the high-order multi-agent systems were addressed in $[24,25]$, where the network was fixed, and it was shown that all the gains must be chosen properly to ensure that consensus could be reached. Consensus regions were discussed for undirected switched topologies in [39]. The consensus of third-order nonlinear multi-agent systems was present in [26] recently, and a consensus protocol was proposed under the assumption that the topology was fixed. However, due to the loss or creation of the link, the topologies would be changed. The studies for consensus under switching topologies were more practical [42]. The consensus problem for high-order dynamic multi-agent systems with fixed and switching topologies was discussed in [41], and sufficient conditions of the consensus for switching topologies were provided under the assumption that interactions among agents were modeled by undirected graphs. A decentralized high gain control law was provided in [40] to solve the consensus problems with undirected switching topologies. But the consensus analysis for directed networks was more challenging than the case of undirected networks [5]. The consensus analysis for third-order multi-agent systems in directed networks was studied in [27] recently, where the topology was fixed. The relationship between high-order integral multi-agent systems and the first-order integral multi-agent systems was researched in [42] recently. For the directed switching networks, even with the unfixed leader, few studies have been done on the general consensus issues when including different kinds of unconsensusable topologies, although a consensus could be reached if the dwell time of consensusable topologies was long enough.

Motivated by the above discussion, consensus analysis by a topology-dependent average dwell time approach, and three kinds of switching were studied in this paper. The main contributions of this paper were reflected as follows: (1) The proposed results in this paper would show the consensus also could be reached under switching topologies including different kinds of unconsensusable topologies. Both improper coupling strengths and no spanning tree were applicable to this case. (2) The present results could be applied to the following cases: no leader; switching leaders; switching between with and without a leader. (3) The results could be suitable for both the balanced network, with fixed group decision value, and the unbalanced network, with changing group decision value as topologies changing. 
The rest of this paper is organized as follows: In Section 2, some preliminaries regarding the concepts of algebraic graph theories, dwell time approaches and definitions are presented. Section 3 details the model transformation and consensus analysis. Numerical examples are made to verify the theoretical analysis in Section 4. Finally, the conclusions are given in Section 5.

Notations: If $P$ is a matrix, $P>(<) 0$ implies that $P$ is symmetric and positive(negative) definite. The operator $\otimes$ represents the Kronecker product of two matrices. Let $I_{c}$ be the $c \times c$ identity matrix, and $\mathbf{0}_{c \times d}$ be the $c \times d$ zero matrix. The superscript "T" stands for the matrix transpose. $C^{1}$ stands for the space of continuously differentiable functions. $\mathbb{R}^{N}$ denotes $\mathrm{N}$-dimensional Euclidean space. If a function $\beta:[0, \infty) \rightarrow[0, \infty), \beta(0)=0$, is strictly increasing, continuous, unbounded, then it is said to be of class $K_{\infty}$. And $t_{1}, t_{2}, t_{3}, \cdots, t_{i}, t_{i+1}, \cdots$ are the switching times.

\section{Preliminaries}

Graph theory is used to model the directed interaction networks among agents in the multi-agent systems with n agents. A digraph is denoted by $\mathcal{G}=(\mathcal{V}, \mathcal{E}, \mathcal{A})$ with a set of nodes $\mathcal{V}=\left\{v_{1}, v_{2}, \ldots, v_{n}\right\}$, a set of edges $\mathcal{E} \subseteq \mathcal{V} \times \mathcal{V}$, and an adjacency matrix $\mathcal{A}=\left[a_{i j}\right]$. The set of node indexes is $\mathcal{I}=\{1,2, \ldots, n\}$. An edge of graph $\mathcal{G}$ is denoted by $e_{i j}=\left(v_{i}, v_{j}\right)$. We assume that there are no self loops. The set of neighbors of node $v_{i}$ is denoted by $\mathcal{N}_{i}=\left\{v_{i} \in \mathcal{V}:\left(v_{i}, v_{j}\right) \in \mathcal{E}, j \neq i\right\}$. A directed tree is a special directed graph, where every node has exactly one parent except the root. A spanning tree of a directed graph is a directed tree that connects all the nodes of the graph. The Laplacian matrix $L$ is defined as: $l_{i j}=\sum_{k=1, k \neq i}^{n} a_{i k}$ for $\mathrm{i}=\mathrm{j}$, and $l_{i j}=-a_{i j}$, for $i \neq j, i, j \in \mathcal{I}$. More details are in [28].

Lemma 1. [6] If the directed network has a directed spanning tree, the corresponding Laplacian matrix $L$ would have a simple eigenvalue 0 and all the other eigenvalues would have positive real parts.

Lemma 2. Let $L=\left[l_{i j}\right] \in \mathbb{R}^{n \times n}$ denote the Laplacian matrix associated with a digraph having a spanning tree. All the non-zero eigenvalues of $L$ are the eigenvalues of the matrix $H=\left[h_{i j}\right] \in$ $\mathbb{R}^{(n-1) \times(n-1)}$, where $h_{i j}=l_{i j}-l_{n j}$. 
Proof. For a strongly connected digraph, this lemma was given in [29] [30]. From lemma 1, if a directed network has a directed spanning tree, 0 also is a simple eigenvalue. The rest part of this proof is similar to that in [29], and we thus omit it here.

Consider a team of $\mathrm{n}$ agents systems where each agent is modeled with $G^{\text {th }}$-order dynamics as follows [24] [25]:

$$
\begin{gathered}
\dot{x}_{i}^{(0)}(t)=x_{i}^{(1)}(t) \\
\vdots \\
\dot{x}_{i}^{(G-2)}(t)=x_{i}^{(G-1)}(t) \\
\dot{x}_{i}^{(G-1)}(t)=u_{i}, i \in \mathcal{I}
\end{gathered}
$$

Where $x_{i}(t)=\left[x_{i 1}(t), \ldots, x_{i N}(t)\right]^{T} \in \mathbb{R}^{N}$ is the state of vector of the $i^{t h}$ agent. $u_{i} \in \mathbb{R}^{N}$ is the control input, and $x_{i}^{(k)}$ denotes the $k^{\text {th }}$ derivative of $x_{i}$ with $x_{i}^{(0)}=x_{i}$

The consensus algorithm is proposed as [20] [24]:

$$
\begin{aligned}
u_{i}(t) & =\beta_{0} \sum_{j \in \mathcal{N}_{\mathrm{i}}(\mathrm{t})} a_{i j}(t)\left(x_{j}^{(0)}(t)-x_{i}^{(0)}(t)\right) \\
& +\cdots \\
& +\beta_{G-2} \sum_{j \in \mathcal{N}_{\mathrm{i}}(\mathrm{t})} a_{i j}(t)\left(x_{j}^{(G-2)}(t)-x_{i}^{(G-2)}(t)\right) \\
& +\beta_{G-1} \sum_{j \in \mathcal{N}_{\mathrm{i}}(\mathrm{t})} a_{i j}(t)\left(x_{j}^{(G-1)}(t)-x_{i}^{(G-1)}(t)\right), i=\mathcal{I}
\end{aligned}
$$

Where $a_{i j}, i, j \in \mathcal{I}$ are the entries of adjacency matrix $\mathcal{A}(\mathcal{G})$, given by the interaction topology $\mathcal{G}$. Positive constant $\beta_{0}, \beta_{1}, \beta_{2}, \ldots, \beta_{G-1}$ are the coupling strengths. And $\mathcal{K}=\{0, \ldots, G-1\}$.

Definition 1. $G^{\text {th }}$-order consensus is said to be reached among $n$ agents for the multi-agent systems if for all initial conditions, $x_{i}^{(k)}(t) \rightarrow x_{j}^{(k)}(t)$, as $t \rightarrow+\infty, k \in \mathcal{K}$. In this paper, we would set $\mathrm{j}$ as n.

In order to solve the consensus problem of multi-agent systems, some theories about switched systems would be used in this paper. Some basic concepts and results of switched systems are introduced. 
Definition 2. [31] For a switching $\sigma(t)$ and any $\mathrm{T} \geq \mathbf{t} \geq 0$. The switching numbers of $p^{\text {th }}$ subsystem is denoted by $N_{\sigma p}(T, t)$, and the total running time of $p^{t h}$ subsystem is denoted by $T_{p}(T, t)$ over the interval [t, T]. $\sigma(t)$ is said to have a mode-dependent average dwell time (MDADT) $\tau_{a p}$ if there exist positive numbers $N_{0 p}(T, t)$ and $\tau_{a p}$ such that

$$
N_{\sigma p}(T, t) \leq N_{0 p}(T, t)+\frac{T_{p}(T, t)}{\tau_{a p}}
$$

Consider the following switched system:

$$
\dot{z}(t)=w_{\sigma(t)}(z(t)), \mathrm{z}\left(t_{0}\right)=z_{0}, t \geq t_{0}
$$

Where $z(t) \in \mathbb{R}^{G(n-1)}$ is another state vector, $\sigma(t)$ is a switching signal. The value of $\sigma(t)$ is in the finite set $\mathcal{J}=\{1, \ldots, M\}$, while $M$ is the number of subsystems. In this paper, we suppose that there are $\mathrm{M}$ subsystems including M-r unstable subsystems. For brevity, the $1^{\text {st }}, 2^{\text {nd }}, \ldots, r^{\text {th }}$ subsystem are stable, denoted as $\mathcal{S}$, while the $(r+1)^{t h}, \ldots, M^{\text {th }}$ are unstable, denoted as $\mathcal{U}$. Suppose $r \geq 1$. It is not known whether $r=M$ or not.

Lemma 3. [31] Consider the switched system (4), and give constants $\lambda_{P}>0, \mu_{P}>1, p \in \mathcal{J}$. Suppose that there exist $C^{1}$ functions $V_{\sigma(t)}: \mathbb{R}^{G(n-1)} \rightarrow \mathbb{R}^{1}$, and class $K_{\infty}$ functions $k_{1 p}$ and $k_{2 p}$, such that $\forall p \in \mathcal{J}$,

$$
\begin{gathered}
k_{1 p}(\|z(t)\|)<V_{p}(\|z(t)\|)<k_{2 p}(\|z(t)\|) \\
\dot{V}_{p}(\|z(t)\|)<-\lambda_{p} V_{p}(\|z(t)\|)
\end{gathered}
$$

and $\forall\left(\sigma\left(t_{i}\right)=p, \sigma\left(t_{i}^{-}\right)=q\right) \in \mathcal{J} \times \mathcal{J}, \mathrm{p} \neq \mathrm{q}$

$$
V_{p}\left(z\left(t_{i}\right)\right)<\mu_{p} V_{q}\left(z\left(t_{i}^{-}\right)\right)
$$

Then the system (4) is globally uniformly asymptotically stable with MDADT

$$
\tau_{a p} \geq \tau_{a p}^{*}=\frac{\ln \mu_{p}}{\lambda_{p}}
$$


Lemma 4. [32] Consider switched system (4), and give constants $\alpha_{P}, \mu_{P}>1, p \in \mathcal{J}$. Suppose that there exist $C^{1}$ functions $V_{\sigma(t)}: \mathrm{R}^{G(n-1)} \rightarrow \mathrm{R}^{1}$, and class $K_{\infty}$ functions $k_{1 p}$ and $k_{2 p}$, such that $\forall p \in \mathcal{J}$,

$$
\begin{gathered}
k_{1 p}(\|z(t)\|)<V_{p}(\|z(t)\|)<k_{2 p}(\|z(t)\|) \\
\dot{V}_{p}(\|z(t)\|)<\alpha_{p} V_{p}(\|z(t)\|)
\end{gathered}
$$

and $\forall\left(\sigma\left(t_{i}\right)=p, \sigma\left(t_{i}^{-}\right)=q\right) \in \mathcal{J} \times \mathcal{J}, \mathrm{p} \neq \mathrm{q}$

$$
V_{p}\left(e\left(t_{i}\right)\right)<\mu_{p} V_{q}\left(e\left(t_{i}^{-}\right)\right)
$$

Then the system is globally uniformly asymptotically stable with marginal $\gamma^{*}$ under any switching signal with the following conditions:

$$
\begin{cases}\tau_{a p} \geq-\frac{\ln \mu_{p}}{\alpha_{p}} & \left(\alpha_{p}<0, p \in \mathcal{S}\right) \\ \tau_{a p} \geq \tau_{a p}^{*} & \left(\alpha_{p}>0, \forall \tau_{a p}^{*}>0, p \in \mathcal{U}\right) \\ \frac{T^{-}}{T^{+}}>\frac{\gamma^{+}+\gamma^{*}}{\gamma^{-}-\gamma^{*}} & \left(0<\gamma^{*}<\gamma^{-}\right)\end{cases}
$$

where $T^{-}=\sum_{p=1}^{r} T_{p}(t, 0), T^{+}=\sum_{p=r+1}^{M} T_{p}(t, 0),-\gamma^{-}=\max _{p \in \mathcal{S}}\left(\alpha_{p}+\frac{\ln \mu_{p}}{\tau_{a p}}\right), \gamma^{+}=\max _{p \in \mathcal{U}}\left(\alpha_{p}+\right.$ $\left.\frac{\ln \mu_{p}}{\tau_{a p}}\right)$.

\section{Main results}

In this section, the model transformation and consensus analysis are studied under switching topologies.

Let $x^{(k)}(t)=\left[x_{1}^{(k)}(t)^{T}, \ldots, x_{n}^{(k)}(t)^{T}\right]^{T}$, and $y(t)=\left[x^{(0)}(t)^{T}, \ldots, x^{(G-1)}(t)^{T}\right]^{T}, k \in \mathcal{K}$. Then, network (1) with input (2) can be rewritten in a compact matrix as

$$
\dot{y}(t)=\left(\tilde{L}(t) \otimes I_{N}\right) y(t)
$$


where

$$
\begin{aligned}
& \tilde{L}(t)=\left[\begin{array}{cc}
\mathbf{0}_{((G-1) n) \times n} & I_{((G-1) n) \times((G-1) n)} \\
-\beta_{0} L(t) & \hat{L}(t)
\end{array}\right]_{(G n) \times(G n)} \\
& \hat{L}(t)=\left[\begin{array}{llll}
-\beta_{1} L(t) & -\beta_{2} L(t) \quad \cdots \quad-\beta_{G-1} L(t)
\end{array}\right]_{n \times((G-1) n)}
\end{aligned}
$$

and $y(t) \in \mathbb{R}^{G N n}, L=\left[l_{i j}\right]$ is the Laplacian matrix.

The $x_{i}^{(k)} \in \mathbb{R}^{N}, x_{i}^{(k)}$ can be denoted as

$$
x_{i}^{(k)}(t)=\left[x_{i, 1}^{(k)}(t), x_{i, 2}^{(k)}(t), \ldots, x_{i, N}^{(k)}(t)\right]^{T}
$$

where $x_{i, j}^{(k)} \in \mathbb{R}^{1}, i \in \mathcal{I}, j \in\{1, \ldots, N\}$.

Let $y_{j}(t)=\left[x_{1, j}^{(0)}(t), x_{2, j}^{(0)}(t), \cdots, x_{n, j}^{(0)}(t), x_{1, j}^{(1)}(t), \cdots, x_{n, j}^{(G-1)}(t)\right]^{T}, j \in\{1, \cdots N\}$, the system (13) could be rewritten as

$$
\left\{\begin{array}{c}
\dot{y}_{1}(t)=\tilde{L} * y_{1}(t), y_{1}(0)=\left.y_{1}(t)\right|_{t=0} \\
\ldots \\
\dot{y}_{N}(t)=\tilde{L} * y_{N}(t), y_{N}(0)=\left.y_{N}(t)\right|_{t=0}
\end{array}\right.
$$

with $\mathrm{N}$ independent subsystems. These $\mathrm{N}$ independent subsystems have a same differential equation of motion as

$$
\dot{c}(t)=\tilde{L}(t) * c(t)
$$

where $c(t) \in \mathbb{R}^{G n}$ stands for $y_{j}, j \in\{1, \cdots N\}$, defined as

$$
\begin{gathered}
c(t)=\left[c^{(0)}(t)^{T}, \ldots, c^{(G-1)}(t)^{T}\right]^{T} \\
c^{(k)}(t)=\left[c_{1}^{(k)}(t), \ldots, c_{n}^{(k)}(t)\right]^{T}, k \in \mathcal{K}
\end{gathered}
$$

And, $c(0)=\left.c(t)\right|_{t=0}$ takes its values from $\left\{\left.y_{j}(t)\right|_{t=0}\right\}, j \in\{1, \ldots, N\}$.

Now the consensus problem with (13) for all initial values is equivalent to that with equation (17) for all initial conditions. Similar analysis was in [24] with fixed topologies. If we assume N=1 
for simplicity, (17) is (13).

By setting $j$ as $n$ for definition 1 , and using(17), $G^{t h}$-order consensus would be said to be reached if for all initial conditions, $c_{i}^{(k)}(t) \rightarrow c_{n}^{(k)}(t)$, as $t \rightarrow+\infty, k \in \mathcal{K}, \forall i \in\{1, \ldots, n-1\}$.

I.e., the consensus problem would be solved if

$$
c_{i}^{(k)}(t)-c_{n}^{(k)}(t) \rightarrow 0, \text { as } t \rightarrow+\infty, \forall k \in \mathcal{K}, \forall \mathrm{i} \in\{1, \ldots, \mathrm{n}-1\}
$$

for all initial conditions.

$\forall k \in \mathcal{K}, \forall \mathrm{i} \in\{1, \ldots, \mathrm{n}-1\}$, let

$$
\left\{\begin{array}{l}
z_{i}^{(k)}(t)=c_{i}^{(k)}(t)-c_{n}^{(k)}(t) \\
z^{(k)}(t)=\left[z_{1}^{(k)}(t)^{T}, \ldots, z_{n-1}^{(k)}(t)^{T}\right]^{T}
\end{array}\right.
$$

and $z(t)=\left[z^{(0)}(t)^{T}, \ldots, z^{(G-1)}(t)^{T}\right]^{T} \in \mathbb{R}^{G(n-1)}$.

Now, one can get the following Lemma 5.

Lemma 5. For the directed network $\mathcal{G}(\mathrm{t})$, if the globally uniformly asymptotically stability problem of system

$$
\dot{z}(t)=\tilde{H}(t) z(t), z\left(t_{0}\right)=z(0) .
$$

is ensured, where

$$
\begin{aligned}
& \tilde{H}(t)=\left[\begin{array}{cc}
\mathbf{0}_{((G-1)(n-1)) \times(n-1)} & I_{((G-1)(n-1)) \times((G-1)(n-1))} \\
-\beta_{0} H(t) & \hat{H}(t)
\end{array}\right]_{(G(n-1)) \times(G(n-1))}
\end{aligned}
$$

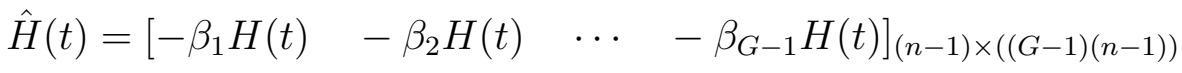

then the consensus of system (1) is reached with consensus algorithm (2), under all initial conditions. 
Proof. The $i^{\text {th }}$ row vector for the Laplacian matrix can be denoted as

$$
L_{i}(t)=\left[l_{i 1}(t), l_{i 2}(t), \ldots, l_{i n}(t)\right], i \in \mathcal{I}
$$

The $i^{t h}$ row vector for the $H$ matrix can be denoted as

$$
H_{i}(t)=\left[h_{i 1}(t), h_{i 2}(t), \ldots, h_{i n-1}(t)\right], i \in \mathcal{I} \quad \text { and } \quad \mathrm{i} \neq \mathrm{n}
$$

1) When $\forall k \in \mathcal{K}$ and $k<G-1$, by (17) and (20), for $\dot{z}_{i}^{(k)}(t)$, then

$$
\begin{aligned}
\dot{z}_{i}^{(k)}(t) & =\dot{c}_{i}^{(k)}(t)-\dot{c}_{n}^{(k)}(t) \\
& =c_{i}^{(k+1)}(t)-c_{n}^{(k+1)}(t), \\
& =z_{i}^{(k+1)}(t)
\end{aligned}
$$

and

$$
\begin{aligned}
\dot{z}^{(k)}(t) & =\left(\dot{z}_{1}^{(k)}(t)^{T}, \ldots, \dot{z}_{n-1}^{(k)}(t)^{T}\right)^{T} \\
& =\left(z_{1}^{(k+1)}(t)^{T}, \ldots, z_{n-1}^{(k+1)}(t)^{T}\right)^{T} \\
& =z^{(k+1)}(t)
\end{aligned}
$$

2) For $\dot{z}_{i}^{(G-1)}(t)$, by (17) and (20), with properties of the Laplacian matrix , then

$$
\begin{aligned}
\dot{z}_{i}^{(G-1)}(t) & =\dot{c}_{i}^{(G-1)}(t)-\dot{c}_{n}^{(G-1)}(t) \\
& =\beta_{0} \sum_{j \in \mathcal{N}_{\mathrm{i}}(\mathrm{t})} a_{i j}(t)\left(c_{j}^{(0)}(t)-c_{i}^{(0)}(t)\right)+\cdots \\
& +\beta_{G-1} \sum_{j \in \mathcal{N}_{\mathrm{i}}(\mathrm{t})} a_{i j}(t)\left(c_{j}^{(G-1)}(t)-c_{i}^{(G-1)}(t)\right) \\
& -\beta_{0} \sum_{j \in \mathcal{N}_{\mathrm{n}}(\mathrm{t})} a_{i j}(t)\left(c_{j}^{(0)}(t)-c_{n}^{(0)}(t)\right)-\cdots \\
& -\beta_{G-1} \sum_{j \in \mathcal{N}_{\mathrm{n}}(\mathrm{t})} a_{i j}(t)\left(c_{j}^{(G-1)}(t)-c_{n}^{(G-1)}(t)\right)
\end{aligned}
$$


By (24), one can get

$$
\begin{aligned}
\dot{z}_{i}^{(G-1)}(t)= & -\beta_{0} L_{i}(t) c^{(0)}(t)-\cdots-\beta_{G-1} L_{i}(t) c^{(G-1)}(t) \\
& +\beta_{0} L_{n}(t) c^{(0)}(t)+\cdots+\beta_{G-1} L_{n}(t) c^{(G-1)}(t) \\
= & -\beta_{0} L_{i}(t) c^{(0)}(t)-\cdots-\beta_{G-1} L_{i}(t) c^{(G-1)}(t) \\
& +\beta_{0} L_{n}(t) c^{(0)}(t)+\cdots+\beta_{G-1} L_{n}(t) c^{(G-1)}(t) \\
= & -\beta_{0}\left(L_{i}(t)-L_{n}(t)\right) c^{(0)}(t)-\cdots \\
& -\beta_{G-1}\left(L_{i}(t)-L_{n}(t)\right) c^{(G-1)}(t)
\end{aligned}
$$

with properties of the Laplacian matrix, and the definition of $H$ matrix in Lemma 2. One can get

$$
\left(L_{i}(t)-L_{n}(t)\right) c^{(k)}(t)=H_{i}(t) z^{(k)}(t), \forall k \in \mathcal{K}
$$

By (29),

$$
\begin{aligned}
\dot{z}_{i}^{(G-1)}(t)= & -\beta_{0}\left(L_{i}(t)-L_{n}(t)\right) c^{(0)}(t)-\cdots \\
& -\beta_{G-1}\left(L_{i}(t)-L_{n}(t)\right) c^{(G-1)}(t) \\
= & -\beta_{0} H_{i}(t) z^{(0)}(t)-\cdots-\beta_{G-1} H_{i}(t) z^{(G-1)}(t)
\end{aligned}
$$

By the definition of $z^{(k)}(t)$ and (25), then

$$
\begin{aligned}
\dot{z}^{(G-1)}(t) & =\left(\dot{z}_{1}^{(G-1)}(t)^{T}, \ldots, \dot{z}_{n-1}^{(G-1)}(t)^{T}\right)^{T} \\
& =-\beta_{0} H(t) z^{(0)}(t)-\cdots-\beta_{G-1} H(t) z^{(G-1)}(t) \\
& =-\beta_{0} H(t) z^{(0)}(t)+\hat{H}\left[z^{(1)}(t)^{T}, \ldots, z^{(G-1)}(t)^{T}\right]^{T}
\end{aligned}
$$

Now, by 1) and 2), the equation (21) has been proved.

If the globally uniformly asymptotically stability problem of system (21) is ensured, then

$$
z_{i}^{(k)}=c_{i}^{(k)}(t)-c_{n}^{(k)}(t) \rightarrow 0 \quad \text { as } \quad t \rightarrow+\infty, \forall k \in \mathcal{K}, \forall \mathrm{i} \in\{1, \ldots, \mathrm{n}-1\}
$$

Compared with consensus definition of equation (20), the consensus problem of (17) is solved. 
Based on the relationship, which has been analyzed, between (17) and (13), the consensus of system (13) is reached. The consensus of system (1) with consensus algorithm (2) is reached, because system (13) is the compact matrix form of system (1) with consensus algorithm (2).

Now one can conclude the proof of this lemma.

Corollary 1. If the directed network $\mathcal{G}$ has a directed spanning tree with fixed topologies, the system (1) with consensus algorithm (2) reach a consensus exponentially if and only if all the eigenvalues of corresponding matrix $\tilde{H}$ have negative real parts.

Proof. Let $\tilde{J}$ be the Jordan form associated with $\tilde{H}$, then $\tilde{H}=S \tilde{J} S^{-1}$. By explicit calculation of (21), one has

$$
z(t)=\exp (\tilde{H} t) z(0)=S \exp (\tilde{J} t) S^{-1} z(0)
$$

$\exp (\tilde{J} t)$ converges to a zero matrix exponentially, as $\mathrm{t} \rightarrow \infty$, if and only if all the eigenvalues of corresponding matrix $\tilde{H}$ have negative real parts.

By the proof of Lemma 5, the systems (21) can be got from the system (1) with consensus algorithm (2), and system (1) with consensus algorithm (2) reaches consensus exponentially if and only if the system (21) converges to origin exponentially, as $t \rightarrow \infty$. The system (21) converges to origin exponentially only if all the eigenvalues of corresponding matrix $\tilde{H}$ have negative real parts, as $\mathrm{t} \rightarrow \infty$.

Now, one can now conclude the proof of the corollary.

Remark 1. The time-variable matrix $H(t)$, like $L(t)$, would change as topologies change, and has all the non-zero eigenvalues of $L(t)$ under the topologies with a spanning tree by lemma 2 . The structure of matrix $\tilde{H}(t)$ is similar to $\tilde{L}(t)$. The matrix $H$ is a Laplacian-like matrix.

Remark 2. By lemma 5, the linear model (21) is time-variable, for the time-variable matrix $H(t)$. The linear model (21) can also be used under switching topologies.

In [20] [24], some certain relations, to coupling strengths with a topology, must be satisfied in order to reach a consensus. Any agent could not know whether the topologies have changed or not, for each agent only using the information from its neighbors. Suppose that the relationships, such 
as coupling strengths, would not change under switching topologies. The multi-agent systems would undergo M kinds of topologies including M-r kinds of topologies that would not make (21) asymptotically stable (the consensus could not be reached). For brevity, the $1^{\text {st }}, 2^{\text {nd }}, \ldots, r^{\text {th }}$ topology are stable, denoted as $\mathcal{S}$, while the $(r+1)^{t h}, \ldots, M^{\text {th }}$ are unstable, denoted as $\mathcal{U}$. Suppose $r \geq 1$. It is not known whether $r=M$ or not. These assumptions are similar to the assumptions with switched systems. Now The mode-dependent average dwell time approach is converted to the topology-dependent average dwell time approach. Some sufficient conditions for consensus would be presented by using the dwell time approach.

Theorem 1. For the multi-agent systems under switching topologies network $\mathcal{G}_{\mathrm{p}}, p \in \mathcal{J}$, if there exist symmetric matrices $\mathrm{P}>0, \forall p \in \mathcal{J}$ such that

$$
\tilde{H}_{p}^{T} P+P \tilde{H}_{p}<\mathbf{0}
$$

Then the multi-agent systems (1) would reach a consensus with consensus algorithm (2) under all initial values.

Proof. A common Lyapunov function is constructed as

$$
V(z(t))=z^{T}(t) P z(t)
$$

and $\forall t \in\left[t_{i}, t_{i+1}\right)$, with (34)

$$
\begin{aligned}
\dot{V}(z(t)) & =\dot{z}^{T}(t) P z(t)+z^{T}(t) P \dot{z}(t) \\
& =z^{T}(t) \tilde{H}_{p}^{T} P z(t)+z^{T}(t) P \tilde{H}_{p} z(t) \\
& =z^{T}(t)\left(\tilde{H}_{p}^{T} P+P \tilde{H}_{p}\right) z(t) \\
& <0
\end{aligned}
$$

the system(21) is globally uniformly asymptotically stability.

By Lemma 5, the multi-agent systems (1) with consensus algorithm (2) would reach a consensus under all initial values. 
This proof is completed.

Remark 3. Both the theorem 1 and corollary 1 can be used under fixed topologies. With condition (34), the multi-agent systems under switching topologies are stable under arbitrary switching. The number of unconsensusable topologies is zero in this case.

In some situations, finding common $P>0$ under condition (34) is difficult, compared to finding an individual $P_{p}>0, \forall p \in \mathcal{J}$,for every topology.

Theorem 2. For the multi-agent systems under switching topologies network $\mathcal{G}_{\mathrm{p}}, p \in \mathcal{J}$, and giving constants $\lambda_{p}>0, \mu_{p}>1, \forall p \in \mathcal{J}$, if there exist symmetric matrices $P_{p}>0, \forall p \in \mathcal{J}$, such that

$$
\tilde{H}_{p}^{T} P_{p}+P_{p} \tilde{H}_{p}<-\lambda_{p} P_{p}
$$

And $\forall\left(\sigma\left(t_{i}\right)=p, \sigma\left(t_{i}^{-}\right)=q\right) \in \mathcal{J} \times \mathcal{J}, \mathrm{p} \neq \mathrm{q}$, such that

$$
P_{p} \leq \mu_{p} P_{q}
$$

Then the multi-agent systems (1) with consensus algorithm (2) and all initial values reach a consensus with

$$
\tau_{a p} \geq \tau_{a p}^{*}=\frac{\ln \mu_{p}}{\lambda_{p}}
$$

Proof. A multiple Lyapunov function is constructed as

$$
V_{p}(z(t))=z^{T}(t) P_{p} z(t)
$$


and $\forall t \in\left[t_{i}, t_{i+1}\right)$, with (37) and (40), one can get

$$
\begin{aligned}
\dot{V}_{p}(z(t)) & =\dot{z}^{T}(t) P_{p} z(t)+z^{T}(t) P_{p} \dot{z}(t) \\
& =z^{T}(t) \tilde{H}_{p}^{T} P_{p} z(t)+z^{T}(t) P_{p} \tilde{H}_{p} z(t) \\
& =z^{T}(t)\left(\tilde{H}_{p}^{T} P_{p}+P_{p} \tilde{H}_{p}\right) z(t) \\
& <-\lambda_{p} z^{T}(t) P_{p} z(t) \\
& =-\lambda_{p} V_{p}(z(t))
\end{aligned}
$$

From (38) and (40), then

$$
V_{p}\left(z\left(t_{i}\right)\right)=z^{T}\left(t_{i}\right) P_{p} z\left(t_{i}\right)<\mu_{p} z^{T}\left(t_{i}^{-}\right) P_{q} z\left(t_{i}^{-}\right)=\mu_{p} V_{q}\left(z\left(t_{i}^{-}\right)\right)
$$

By Lemma 3, the system (21) is globally uniformly asymptotically stability with (39).

By Lemma 5, the multi-agent systems (1) with consensus algorithm (2) would reach a consensus with (39).

This proof is completed.

If the number of unconsensusable topologies is more than zero, finding an individual $P_{p}>0$, satisfying (39), $\forall p \in \mathcal{J}$,for every topology is impossible. The following theorem 3 is used for the consensus problem of this situation.

Theorem 3. For the multi-agent systems under switching topologies network $\mathcal{G}_{\mathrm{p}}, p \in \mathcal{J}$, and giving constants $\alpha_{p}, \mu_{p}>1, \forall p \in \mathcal{J}$, if there exist symmetric matrices $P_{p}>0, \forall p \in \mathcal{J}$, such that

$$
\tilde{H}_{p}^{T} P_{p}+P_{p} \tilde{H}_{p}<\alpha_{p} P_{p}
$$

And $\forall\left(\sigma\left(t_{i}\right)=p, \sigma\left(t_{i}^{-}\right)=q\right) \in \mathcal{J} \times \mathcal{J}, \mathrm{p} \neq \mathrm{q}$, such that

$$
P_{p} \leq \mu_{p} P_{q}
$$

Then the multi-agent systems (1) with consensus algorithm (2) and all initial values reach a con- 
sensus with

$$
\begin{cases}\tau_{a p} \geq-\frac{\ln \mu_{p}}{\alpha_{p}} & \left(\alpha_{p}<0, p \in \mathcal{S}\right) \\ \tau_{a p} \geq \tau_{a p}^{*} & \left(\alpha_{p}>0, \forall \tau_{a p}^{*}>0, p \in \mathcal{U}\right) \\ \frac{T^{-}}{T^{+}}>\frac{\gamma^{+}+\gamma^{*}}{\gamma^{-}-\gamma^{*}} & \left(0<\gamma^{*}<\gamma^{-}\right)\end{cases}
$$

where $T^{-}=\sum_{p=1}^{r} T_{p}(t, 0), T^{+}=\sum_{p=r+1}^{M} T_{p}(t, 0),-\gamma^{-}=\max _{p \in S}\left(\alpha_{p}+\frac{\ln \mu_{p}}{\tau_{a p}}\right), \gamma^{+}=\max _{p \in U}\left(\alpha_{p}+\right.$ $\left.\frac{\ln \mu_{p}}{\tau_{a p}}\right)$

Proof. A multiple Lyapunov function is constructed as

$$
V_{p}(z(t))=z^{T}(t) P_{p} z(t)
$$

and $\forall t \in\left[t_{i}, t_{i+1}\right)$, with (43) and (46), one can get

$$
\begin{aligned}
\dot{V}_{p}(z(t)) & =\dot{z}^{T}(t) P_{p} z(t)+z^{T}(t) P_{p} \dot{z}(t) \\
& =z^{T}(t) \tilde{H}_{p}^{T} P_{p} z(t)+z^{T}(t) P_{p} \tilde{H}_{p} z(t) \\
& =z^{T}(t)\left(\tilde{H}_{p}^{T} P_{p}+P_{p} \tilde{H}_{p}\right) z(t) \\
& <\alpha_{p} z^{T}(t) P_{p} z(t) \\
& =\alpha_{p} V_{p}(z(t))
\end{aligned}
$$

From (44) and (46), then

$$
V_{p}\left(z\left(t_{i}\right)\right)=z^{T}\left(t_{i}\right) P_{p} z\left(t_{i}\right)<\mu_{p} z^{T}\left(t_{i}^{-}\right) P_{q} z\left(t_{i}^{-}\right)=\mu_{p} V_{q}\left(z\left(t_{i}^{-}\right)\right)
$$

By Lemma 3, the system(21) is globally uniformly asymptotically stability with (45).

By Lemma 5, the multi-agent systems (1) with consensus algorithm (2) would reach a consensus with(45).

This proof is completed.

Remark 4. In [20], $\mathrm{G}=2$, group decision value $x_{i}^{(1)} \rightarrow \sum_{j=1}^{n} \xi_{j} x_{j}^{(1)}(0), x_{i}^{(0)} \rightarrow \sum_{j=1}^{n} \xi_{j} x_{j}^{(1)}(0) t+$ 
$\sum_{j=1}^{n} \xi_{j} x_{j}^{(0)}(0)$, as as $\mathrm{t} \rightarrow \infty$, where $\xi$, satisfying $\xi^{T} 1_{n}=1$, is the unique nonnegative left eigenvector of $L$ associated with eigenvalue 0 . Group decision value would change as topologies change, even with the same initial values. This class of problems could be solved by the results in this paper.

Remark 5. The results and methods in this paper are also suitable for the consensus algorithm (2) with states as [41], $u_{i}(t)=-\sum_{k=1}^{G-1} \beta_{k} x_{j}^{(k)}-\beta_{0} \sum_{j \in \mathcal{N}_{\mathrm{i}}(\mathrm{t})} a_{i j}(t)\left(x_{i}^{(0)}-x_{j}^{(0)}\right)$. In this case, system (15) is $\hat{L}(t)=\left[-\beta_{1} I-\beta_{2} I \cdots-\beta_{G-1} I\right]$; system (23) is $\hat{H}(t)=\left[-\beta_{1} I-\beta_{2} I \cdots-\beta_{G-1} I\right]$. The new consensus algorithm (2) has no influence on the analysis process in this paper. The results in this paper still hold.

\section{Simulation examples}

In this section, three simulations are given to verify the theoretical analysis. The multi-agent systems with $n=4, N=1, G=2$, under the switching topologies shown in Fig.1, would be considered. The digraph models considered here partly come from [20]. For the following example 1 and example 2, the three-level signal values (low, middle and high) of switching signal $\sigma(t)$ stand for these three topologies((a),(b) and (c)) in Fig.1. For the following example 3, four level signal values from low to high stand for topologies ((a),(b), (c) and (d)) in Fig.1.

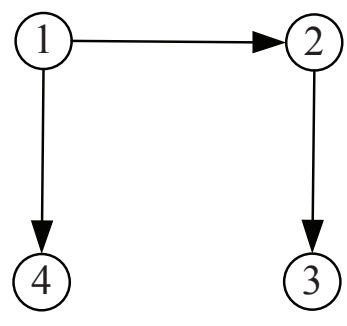

(a)

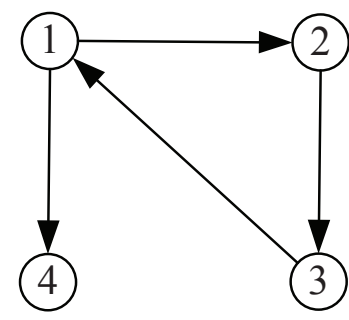

(b)
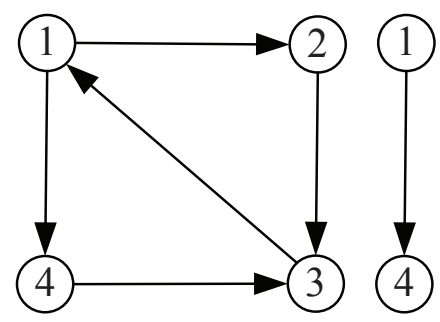

(c)

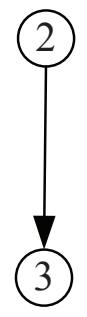

(d)

\section{Fig. 1. Communication network digraphs}

In Fig.1(a), the No.1 agent has no neighbors, and its information can be received by the No.2 agent and the No.4 agent. The No.1 agent could be seen as a leader under the topology in Fig.1(a). There is no agent which has no neighbors and can send its own information to others in Fig.1(b) and Fig.1(c). The switching under these topologies (Fig.1(a-c)) can be considered as the switching 
between with and without a leader.

The adjacency matrixes of the topologies in Fig.1 (a-c) are $\left[\begin{array}{cccc}0 & 0 & 0 & 0 \\ 1 & 0 & 0 & 0 \\ 0 & 1 & 0 & 0 \\ 1 & 0 & 0 & 0\end{array}\right],\left[\begin{array}{cccc}0 & 0 & 1 & 0 \\ 1 & 0 & 0 & 0 \\ 0 & 1 & 0 & 0 \\ 1 & 0 & 0 & 0\end{array}\right]$, and $\left[\begin{array}{cccc}0 & 0 & 1 & 0 \\ 1 & 0 & 0 & 0 \\ 0 & 1 & 0 & 1 \\ 1 & 0 & 0 & 0\end{array}\right]$.

They are all unbalanced network by the definition in [5]. The three group decision values under these three topologies are different from each other by the result in [20] with same initial values.

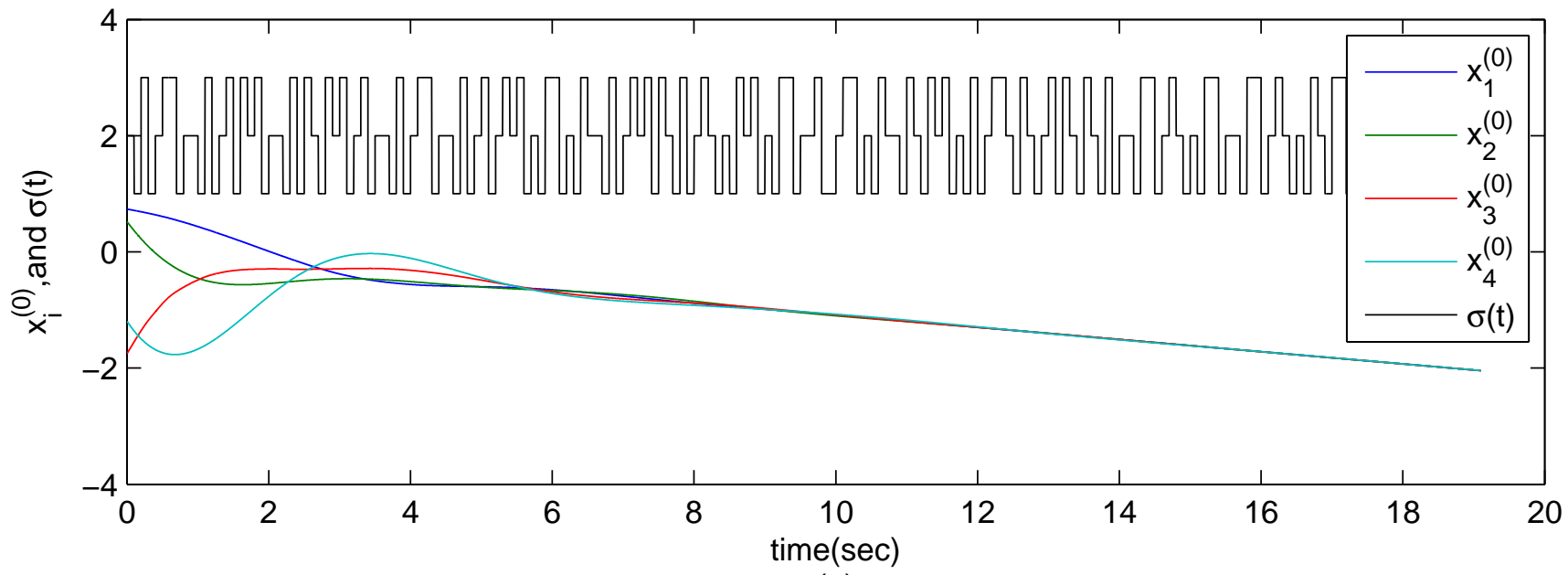

(a)

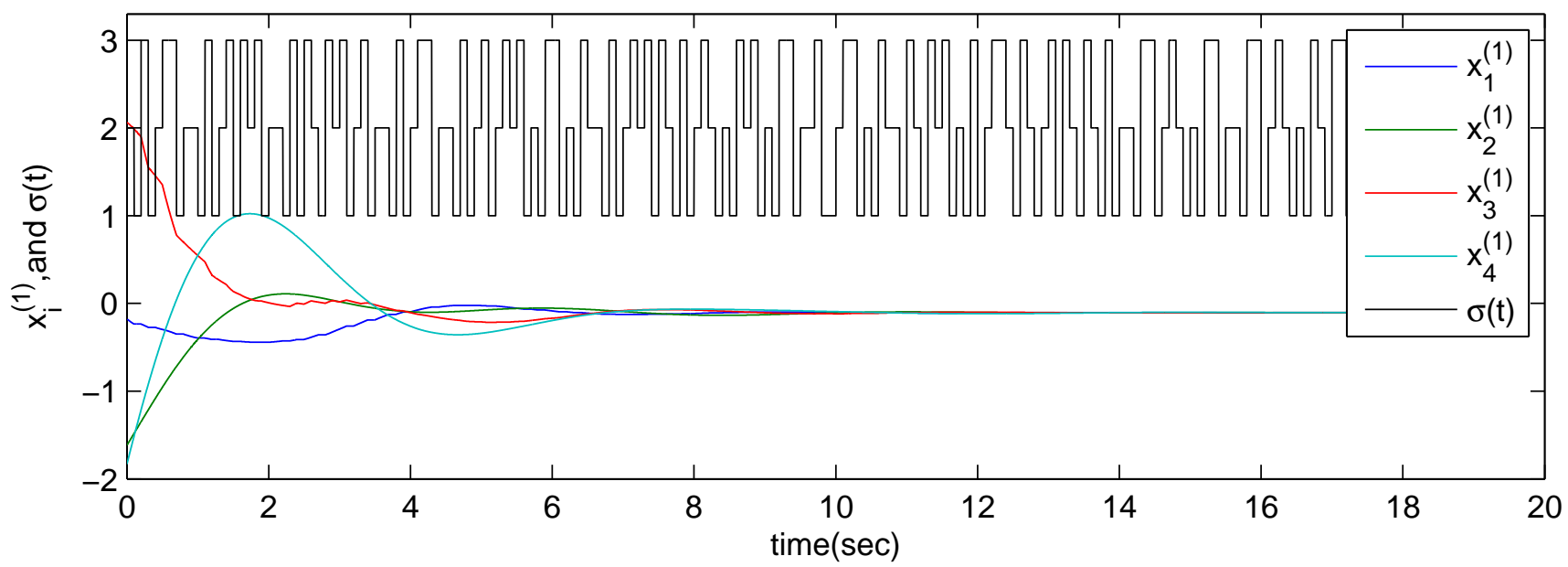

(b)

Fig. 2. States of agents and switching signal, where $\beta_{1}=0.7950$ a Position states b Velocity states 
The coupling strengths, $\beta_{0}$ and $\beta_{1}$, would not change under switching topologies. For simplicity, $\beta_{0}$ is always set 1 in following three examples. In order to reach a consensus with all initial values, $\beta_{1}$ must be more than $0,0.4082$ and 0.3162 by [20], under these three fixed topologies in Fig.1(ac). They are unconsensusable topologies otherwise. In order to verify the main results in the paper, $\beta_{1}$ would be given three different values in following three examples.

Example 1. Firstly, the theorem 1 is verified. When $\beta_{1}=0.7950$, the MATLAB-based toolbox for linear matrix inequality (LMI) could find a common positive definite symmetric matrix $P>0$ satisfying (34), as

$$
\left[\begin{array}{rrrrrr}
7.6950 & -1.8876 & -0.6155 & 2.1487 & 0.7856 & -0.2723 \\
-1.8876 & 15.3995 & -0.6974 & -2.0894 & 5.6901 & 1.4863 \\
-0.6155 & -0.6974 & 3.9337 & -1.2689 & -1.7589 & 1.5151 \\
2.1487 & -2.0894 & -1.2689 & 7.1153 & -3.0718 & -1.5474 \\
0.7856 & 5.6901 & -1.7589 & -3.0718 & 14.0173 & 0.9295 \\
-0.2723 & 1.4863 & 1.5151 & -1.5474 & 0.9295 & 2.9734
\end{array}\right]
$$

In this case, by theorem 1 in this paper, Lyapunov function is strictly non-increasing, and a consensus can reached with all initial values for any arbitrary switching sequences. The states of all agents and switching signals are shown in Fig.2. The first-order states (position states) and secondorder states (velocity states) of the same agent are plotted in same color. A consensus is reached as shown in Fig.2 under switching topologies.

Example 2. Secondly, the theorem 2 is verified. When $\beta_{1}=0.5100$, the LMI toolbox could not find a common positive definite symmetric matrix P satisfying (34). While given constant values $\lambda_{1}=0.0100, \lambda_{2}=0.1900, \lambda_{3}=0.4300$, and $\mu_{1}=1.0002, \mu_{2}=2.4800, \mu_{3}=2.5000$, the LMI toolbox, with the aid of these constant values, could find three different positive definite symmetric 


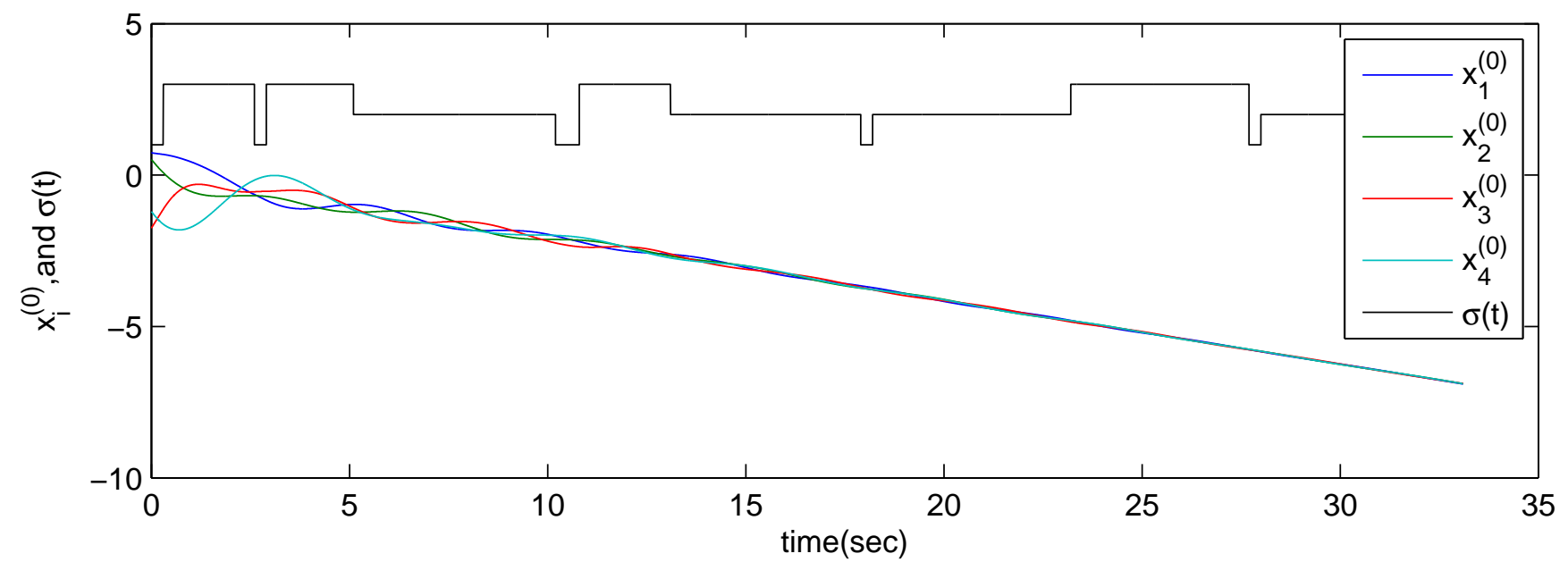

(a)

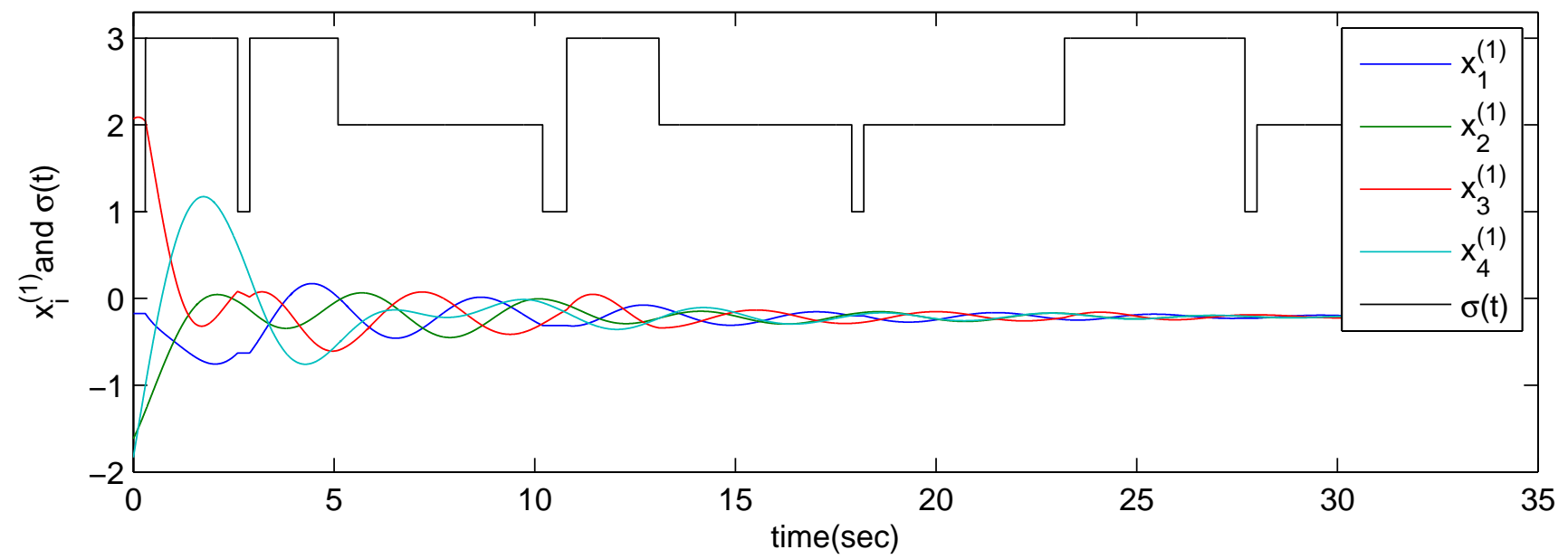

(b)

Fig. 3. States of agents and switching signal, where $\beta_{1}=0.5100$

a Position states

b Velocity states

matrixes, meeting with (37)(38), as

$$
P_{1}=\left[\begin{array}{rrrrrr}
0.0951 & -0.0407 & -0.0071 & 0.0257 & 0.0341 & -0.0117 \\
-0.0407 & 5.8717 & -0.0544 & -0.0465 & 2.2782 & 0.0240 \\
-0.0071 & -0.0544 & 0.0835 & -0.0011 & -0.0193 & 0.0234 \\
0.0257 & -0.0465 & -0.0011 & 0.1193 & -0.0785 & -0.0470 \\
0.0341 & 2.2782 & -0.0193 & -0.0785 & 5.2392 & 0.0218 \\
-0.0117 & 0.0240 & 0.0234 & -0.0470 & 0.0218 & 0.0635
\end{array}\right] * 1000
$$




$$
P_{2}=\left[\begin{array}{rrrrrr}
0.1812 & -0.0697 & -0.0506 & 0.0264 & 0.0495 & -0.0285 \\
-0.0697 & 6.9171 & -0.1093 & -0.0262 & 1.9469 & 0.0031 \\
-0.0506 & -0.1093 & 0.1764 & -0.0302 & -0.0647 & 0.0762 \\
0.0264 & -0.0262 & -0.0302 & 0.1353 & -0.0592 & -0.0755 \\
0.0495 & 1.9469 & -0.0647 & -0.0592 & 6.2723 & -0.0144 \\
-0.0285 & 0.0031 & 0.0762 & -0.0755 & -0.0144 & 0.1201
\end{array}\right] * 1000
$$

By theorem 2 in this paper, a consensus with all initial values would be reached if $\tau_{a 1} \geq 0.0200$, $\tau_{a 2} \geq 4.7803, \tau_{a 3} \geq 2.1309$. The states of all agents and switching signals are shown in Fig.3, where where $\beta_{1}=0.5100$. A consensus would be reached as shown.

Example 3. Thirdly, the theorem 3 is verified. The topology in Fig.1(b) is unconsensusable resulting from improper coupling strengths, when $\beta_{1}=0.3700<0.4082$. The topology in Fig.1(d) is unconsensusable resulting from having no spanning tree. By theorem 3 in this paper, a consensus could also be reached under switching topologies among Fig.1(a-d) including two kinds of unconsensusable topologies. While giving constant values $\alpha_{1}=-0.1000, \alpha_{2}=0.4000, \alpha_{3}=-0.0950$, $\alpha_{4}=0.4700$ and $\mu_{1}=1.0002, \mu_{2}=4.0000, \mu_{3}=4.4500, \mu_{4}=4.7000$, the LMI toolbox could 


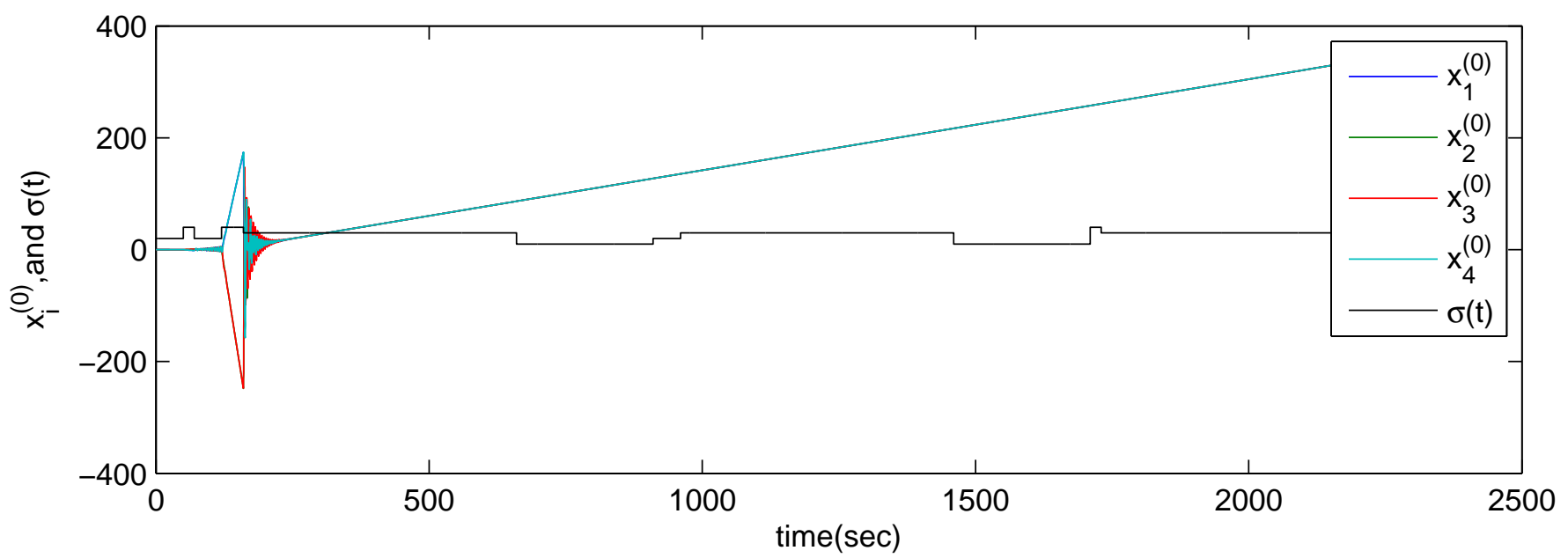

(a)

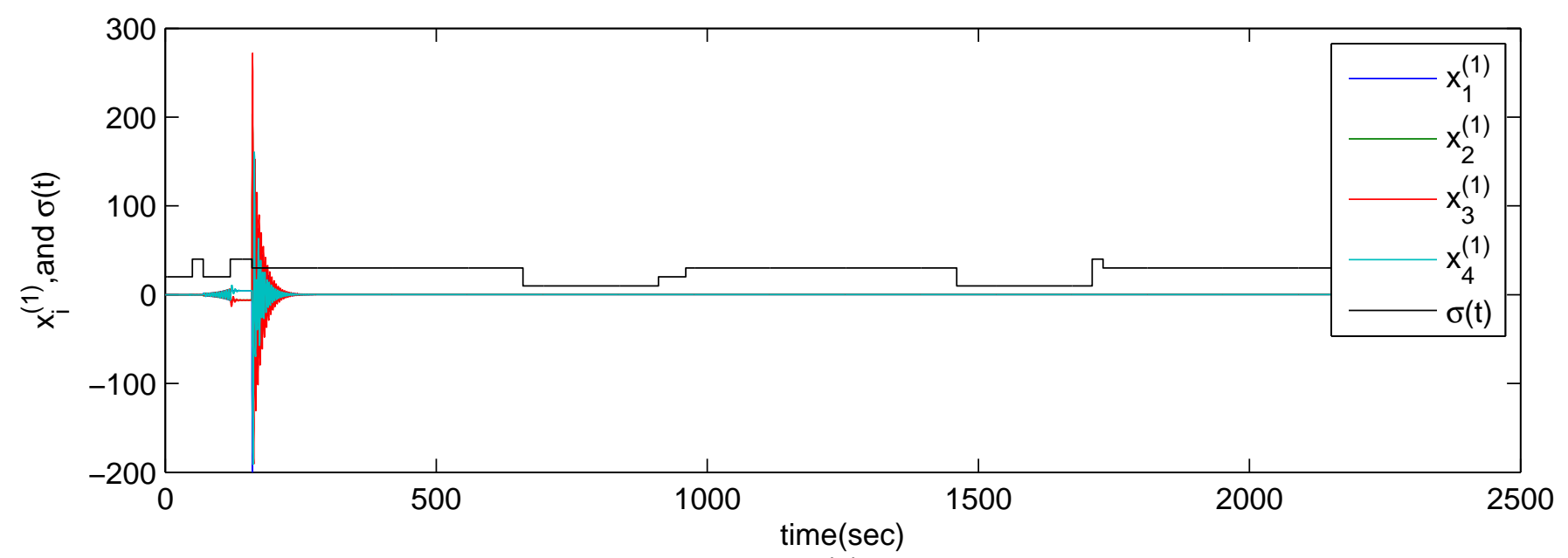

(b)

Fig. 4. States of agents and switching signal, where $\beta_{1}=0.3700$

a Position states

b Velocity states

find four different positive definite symmetric matrixes,, meeting with (43)(44), as

$$
P_{1}=\left[\begin{array}{rrrrrr}
0.1004 & -0.0955 & 0.0126 & 0.0114 & -0.0024 & -0.0029 \\
-0.0955 & 0.1232 & -0.0280 & -0.0073 & 0.0010 & -0.0066 \\
0.0126 & -0.0280 & 0.0301 & 0.0006 & 0.0067 & 0.0071 \\
0.0114 & -0.0073 & 0.0006 & 0.1026 & -0.0940 & -0.0101 \\
-0.0024 & 0.0010 & 0.0067 & -0.0940 & 0.1131 & -0.0010 \\
-0.0029 & -0.0066 & 0.0071 & -0.0101 & -0.0010 & 0.0254
\end{array}\right]
$$




$$
P_{2}=\left[\begin{array}{rrrrrr}
0.1799 & -0.1308 & -0.0130 & 0.0153 & 0.0060 & -0.0218 \\
-0.1308 & 0.1846 & -0.0334 & -0.0051 & -0.0001 & -0.0116 \\
-0.0130 & -0.0334 & 0.0844 & -0.0066 & 0.0166 & 0.0244 \\
0.0153 & -0.0051 & -0.0066 & 0.1159 & -0.0986 & -0.0135 \\
0.0060 & -0.0001 & 0.0166 & -0.0986 & 0.1594 & -0.0123 \\
-0.0218 & -0.0116 & 0.0244 & -0.0135 & -0.0123 & 0.0590
\end{array}\right]
$$

By theorem 3 ,when $\tau_{a 2}=45.0000, \tau_{a 4}=20.0000$, a consensus would be reached if $T^{-} / T^{+}>$ 6.7898, $\tau_{a 1} \geq 0.0020, \tau_{a 1} \geq 15.7184$ under switching topologies. The states of all agents and switching signals are shown in Fig.4, where $\beta_{1}=0.3700$. A consensus is reached as shown.

\section{Conclusion}

In this paper, the consensus problem for multi-agent systems under switching topologies is studied. By a model transformation, the consensus problem under switching topologies is converted into the stability problem of the corresponding switched systems. Based theories about switched systems, 
three theories about consensus are presented. The global consensus with all initial values could be reached under kinds of unconsensusable topologies by these theories. In the future work, we will consider the consensus problem of nonlinear systems under switching topologies.

\section{Acknowledgment}

The work was supported in part by the National Natural Science Foundation of China (Grant no. 61104038, Grant no, 61004048, Grant no. 61174137, Grant no. 61374117 and Grant no. 61374086), the NSF of Jiang Su Province (Grant no. BK2010493), the grant from China Postdoctoral Science Foundation funded project 2012M510135, the Program for Changjiang Scholars and Innovative Research Team in University, the project for science \& technology department of Sichuan Province(Grant no. 2013GZ0080), the 973 project 2011CB707000.

\section{References}

[1] Monostori L., Valckenaers P., Dolgui A., et al.: 'Cooperative control in production and logistics', Annu. Rev. Control , 2015, 39, (1), pp. 12-29

[2] Wang X., Chen M., Kwon T., et al::'Multiple mobile agents' itinerary planning in wireless sensor networks: survey and evaluation', IET Communications, 2011, 5, (12), pp. 1769-1776

[3] Dafflon B., Gechter F., Gruer P., et al.: 'Vehicle platoon and obstacle avoidance: A reactive agent approach', IET Intell. Transp. Syst. , 2013, 7, (3), pp. 257-264

[4] Zhu .B., Liu H., and Li Z.:'Robust distributed attitude synchronization of multiple three-dof experimental helicopters', Control Eng. Practice , 2015, 36, (1), pp. 87-99

[5] Olfati-Saber R., and Murray R. M.:'Consensus problems in networks of agents with switching topology and time-delays', IEEE Trans. Autom. Control ,2004, 49, (9), pp. 1520-1533

[6] Ren W. and Beard R. W.:'Consensus seeking in multiagent systems under dynamically changing interaction topologies', IEEE Trans. Autom. Control , 2005, 50, (5), pp. 655-661 
[7] Yang X. and Liu G.:'Necessary and sufficient consensus conditions of descriptor multi-agent systems', IEEE Trans. Circuils Syst. I. Reg. Papers , 2012, 59, (11), pp. 2669-2677

[8] Han Y., Lu W., and Chen T.:'Achieving cluster consensus in continuous-time networks of multi-agents with inter-cluster non-identical inputs', IEEE Trans. Autom. Control, 2015, 60, (3), pp. 793-798

[9] Chu H., Yuan J., and Zhang W.:'Observer-based adaptive consensus tracking for linear multiagent systems with input saturation', IET Control Theory Appl. , 2015, 9, (14), pp. 2124-2131

[10] Liu H., Cheng L., Tan M., et al.:"Containment control of continuous-time linear multi-agent systems with aperiodic sampling', Automatica, 2015, 57, (1), pp. 78-84

[11] Haghshenas H., Badamchizadeh M. A., and Baradarannia M.:'Containment control of heterogeneous linear multi-agent systems', Automatica, 2015, 54, (1), pp. 210-216

[12] Wen G., Duan Z., Su H., et al.: 'A connectivity-preserving flocking algorithm for multi-agent dynamical systems with bounded potential function', IET Control Theory Appl., 2012, 6, (6), pp. $813-821$

[13] Dong Y. and Huang J.:'A leader-following rendezvous problem of double integrator multiagent systems ', Automatica, 2013, 49, (5), pp. 1386-1391

[14] He F., Wang Y., Yao Y., et al.:'Distributed formation control of mobile autonomous agents using relative position measurements', IET Control Theory Appl. , 2013, 7, (11), pp. 15401552

[15] Ren W., Beard R. W., and Atkins E. M.:'A survey of consensus problems in multi-agent coordination', in Proc. Amer. Contr. Conf, Porland, Jun 2005, pp. 1859-1864.

[16] Sakurama K., Azuma S., and Sugie T.:'Distributed controllers for multi-agent coordination via gradient-flow approach', IEEE Trans. Autom. Control , 2015, 60, (6), pp. 1471-1485

[17] Cao Y., Yu W., Ren W., et al.:'An overview of recent progress in the study of distributed multi-agent coordination', IEEE Trans Ind. Informat. , 2013, 9, (1), pp. 427-438 
[18] Chen Y., Lu J., Yu X., et al.:'Multi-agent systems with dynamical topologies: Consensus and applications', IEEE Circuits Syst. Mag. , 2013, 13, (3), pp. 21-34

[19] Ren W. and Atkins E.:'Second-order consensus protocols in multiple vehicle systems with local interactions', in AIAA Guid.,Navigation Control Conf. Exhibit., San Francisco, USA, 2005, pp. 15-18

[20] Yu W., Chen G., and Cao M.:'Some necessary and sufficient conditions for second-order consensus in multi-agent dynamical systems', Automatica, 2010, 46, (6), pp. 1089-1095

[21] Wen G., Duan Z., Yu W., et al.: 'Consensus in multi-agent systems with communication constraints', Int. J. Robust Nonlinear Control, 2012, 22, (2), pp. 170-182

[22] Abdessameud A. and Tayebi A.:'On consensus algorithms design for double integrator dynamics ', Automatica, 2013, 49, (1), pp. 253-260

[23] Qin J., Gao H., and Zheng W.:'Second-order consensus for multi-agent systems with switching topology and communication delay', Syst. \& Control Lett. , 2011, 60, (6), pp. 390-397

[24] Ren W., Moore K. L., and Chen Y.: 'High-order and model reference consensus algorithms in cooperative control of multivehicle systems', ASME J. Dyn. Syst., Meas., Control, Ft Lauderdale, USA, 2007, 129, (5), pp. 678-688

[25] He W. and Cao J.: 'Consensus control for high-order multi-agent systems', IET Control Theory Appl., 2011, 5, (1), pp. 231-238

[26] Xin Y., Li Y., Huang X., et al.:'Consensus of third-order nonlinear multi-agent systems', Neurocomputing, 2015, 159, (1), pp. 84-89

[27] Cao Y. and Sun Y.:'Consensus analysis for third-order multiagent systems in directed networks', Math. Prob. Eng., 2015, 2015, (1), pp. 1-8

[28] Horn R. A. and Johnson C. R.:'Matrix analysis'(Cambridge university press, 2012) 
[29] Saboori I. and Khorasani K.: 'An $\mathrm{H}_{\infty}$ consensus achievement for LTI multi-agent systems with directed fixed networks', in Proc. 25th IEEE Canadian Conf. Electr. Comp. Eng., Montreal, CANADA, 2012, pp. 1-5

[30] Saboori I. and Khorasani K.: Actuator fault accommodation strategy for a team of multi-agent systems subject to switching topology', Automatica, 2015, 62, (1), pp. 200-207

[31] Zhao X., Zhang L., Shi P., et al.: 'Stability and stabilization of switched linear systems with mode-dependent average dwell time', IEEE Trans. Autom. Control, 2012, 57, (7), pp. 18091815

[32] Xie D., Zhang H., Zhang H., et al.: 'Exponential stability of switched systems with unstable subsystems: A mode-dependent average dwell time approach', Circuits, Syst., Signal Process., 2013, 32, (6), pp. 3093-3105

[33] Hong Y., Hu J., and Gao L.:'Tracking control for multi-agent consensus with an active leader and variable topology'. Automatica,2006, 42, (7), pp.1177-1182

[34] Lin P., and Jia Y.:'Average consensus in networks of multi-agents with both switching topology and coupling time-delay'. Physica A, 2008, 387, (1), pp.303-313

[35] Xiao F., and Wang L.: 'Asynchronous consensus in continuous-time multi-agent systems with switching topology and time-varying delays', IEEE Trans. Autom. Control, 2008, 53, (8), pp.1804-1816

[36] Ni W., and Cheng D.:'Leader-following consensus of multi-agent systems under fixed and switching topologies'. Syst. \& Control Lett., 2010, 59, (3), pp.209-217

[37] Su Y.,and Huang J.: 'Stability of a class of linear switching systems with applications to two consensus problems'. IEEE Trans. Autom. Control, 2012, 57, (6), pp.1420-1430

[38] Li H., Liao X., Huang T., et al.:'Event-triggering sampling based leader-following consensus in second-order multi-agent systems'. IEEE Trans. Autom. Control, 2015, 60, (7), pp.19982003 
[39] Yu W., Chen G., Ren W., et al.:'Distributed higher order consensus protocols in multiagent dynamical systems'. IEEE Trans. Circuils Syst. I. Reg. Papers, 2011, 58, (8), pp.1924-1932

[40] Wang J., Cheng D., and Hu X.:'Consensus of multi-agent linear dynamic systems'. Asian J. Control, 2008, 10, (2), pp.144-155

[41] Jiang F., and Wang L.:'Consensus seeking of high-order dynamic multi-agent systems with fixed and switching topologies'. Int. J. Control, 2010, 83, (2), pp.404-420

[42] Cheng L., Wang H., Hou Z., et al.:'Reaching a consensus in networks of high-order integral agents under switching directed topologies'. Int. J. Syst. Sci., 2016, 47, (8), pp.1966-1981

[43] Wen G., Duan Z., Chen G., et al.:'Consensus Tracking of Multi-Agent Systems with Lipschitz-Type Node Dynamics and Switching Topologies'. IEEE Trans. Circuils Syst. I. Reg. Papers, 2014, 61, (2), pp.499-511 